\title{
Nongenetic Control of Receptor Signaling Dynamics with a DNA-based Optochemical Tool
}

Ryosuke Ueki, Shota Hayashi, Masaya Tsunoda, Momoko Akiyama, Hanrui Liu, Tasuku Ueno, Yasuteru Urano, Shinsuke Sando

Submitted date: 07/01/2020 - Posted date: 08/01/2020

Licence: CC BY-NC-ND 4.0

Citation information: Ueki, Ryosuke; Hayashi, Shota; Tsunoda, Masaya; Akiyama, Momoko; Liu, Hanrui; Ueno, Tasuku; et al. (2020): Nongenetic Control of Receptor Signaling Dynamics with a DNA-based Optochemical Tool. ChemRxiv. Preprint. https://doi.org/10.26434/chemrxiv.11533506.v1

Optochemical tools that can modulate activity of the target protein provide an opportunity for studying and regulating the related biological processes. Here we present a DNA-based nongenetic optochemical tool that can control dynamics of growth factor signaling. A photo-caged DNA aptamer for a growth factor receptor was designed to assemble into an active dimer form, depending on UV irradiation, thereby enabling optical control of the dimerization and subsequent activation of the receptor. This photo-caged mimicry of growth factor can be a promising tool for elucidating a linkage between dynamics of signaling and resulting biological outcomes, as well as for manipulating cellular functions and the fate of living cells.

File list (6)

MT.pdf (1.72 MiB)

SI.pdf (1.64 MiB)

Supporting video 1.avi (1.80 MiB)

Supporting video 2.avi (1.93 MiB)

Supporting video 3.avi (3.22 MiB)

Supporting video 4.avi (2.33 MiB) view on ChemRxiv • download file view on ChemRxiv - download file view on ChemRxiv - download file view on ChemRxiv - download file view on ChemRxiv - download file view on ChemRxiv - download file 


\section{2 chemical Tool}

3 Ryosuke Ueki, ${ }^{* \dagger}$ Shota Hayashi, ${ }^{\dagger}$ Masaya Tsunoda, ${ }^{\dagger}$ Momoko Akiyama, ${ }^{\dagger}$ Hanrui Liu, ${ }^{\dagger}$ Tasuku Ueno,,

4 Yasuteru Urano,

5 tDepartment of Chemistry and Biotechnology and "Department of Bioengineering, The University of 6 Tokyo, 7-3-1 Hongo, Bunkyo-ku, Tokyo, 113-8656, Japan.

7 Graduate School of Pharmaceutical Sciences and ${ }^{\$}$ Graduate School of Medicine, The University of 8 Tokyo, 7-3-1 Hongo, Bunkyo-ku, Tokyo 113-0033, Japan.

ABSTRACT

Optochemical tools that can modulate activity of the target protein provide an opportunity for studying and regulating the related biological processes. Here we present a DNA-based nongenetic optochemical tool that can control dynamics of growth factor signaling. A photo-caged DNA aptamer for a growth factor receptor was designed to assemble into an active dimer form, depending on UV irradiation, thereby enabling optical control of the dimerization and subsequent activation of the receptor. This photo-caged mimicry of growth factor can be a promising tool for elucidating a linkage between dynamics of signaling and resulting biological outcomes, as well as for manipulating cellular functions and the fate of living cells.

\section{MAIN TEXT}

Growth factors (GFs) are polypeptide ligands that regulate various cellular activities such as proliferation, migration, and differentiation. Binding of GFs to receptor tyrosine kinases (RTKs) on the cell membrane induces dimerization and subsequent phosphorylation of the RTKs and initiates phosphorylation cascades of intracellular kinases ${ }^{1}$. Intriguingly, the activation dynamics of kinases downstream of RTKs plays an important role in determining a cellular function and fate (Figure 1) ${ }^{2}$. For example, in rat PC12 cells, both epidermal growth factor (EGF) and nerve growth factor (NGF) activate the Ras-RafMek-Erk pathway via activation of their cognate receptors, but the dynamics of kinase activation and the resulting cellular outcomes show unique patterns ${ }^{3}$. EGF causes transient activation of Erk that leads to cell proliferation, while NGF causes sustained activation of Erk that leads to cell differentiation. To date, much attention has been focused on the mechanisms that initiate such dynamics of GF signaling, thereby regulating cellular functions. Therefore, a method capable of controlling the activity of RTKs 
the dynamics of GF signaling and rewiring a cellular function of living cells by modulating the signaling dynamics ${ }^{4}$.

Optochemical tools that target the interaction between GF and RTK represent a promising approach for the regulation of the dynamics of GF signaling without any gene transfer process. Luebke et al. reported synthesis of EGF containing a photo-caged glutamine at the Glu40 position, which is involved in the interaction with the receptor of EGF, using solid phase peptide synthesis (SPPS) ${ }^{5}$. The photo-caged EGF showed reduced affinity toward the receptor, compared with that of native EGF. A light-irradiation liberated the caging group and restored the binding affinity to the receptor. One intrinsic issue with this approach is the difficulty to find an optimal modification site for the caging group. The photo-caged EGF showed only four-fold higher change in the effective concentration. Therefore, concentration of the ligand should be optimized to achieve low background signal at an expense of high off/on ratio. In addition, this strategy cannot be applied to the majority of GFs, as they consist of large and complex polypeptides with post-translational modifications, which render their preparation via SPPS, challenging and labor-intensive.

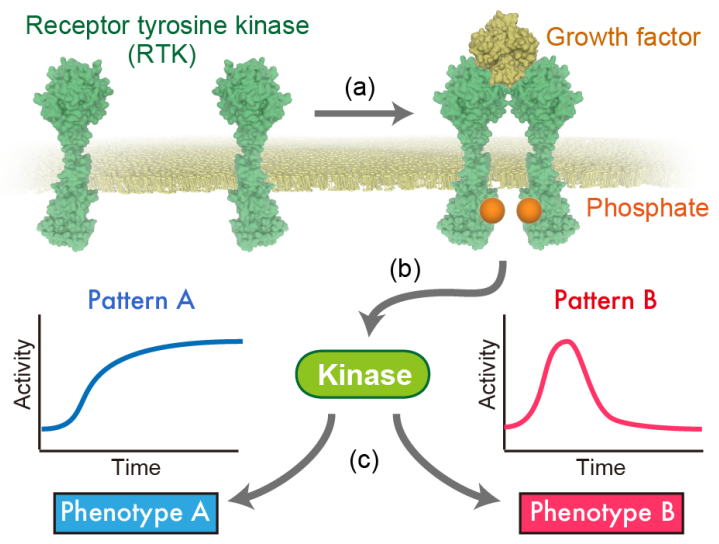

Figure 1. Schematic representation of GF signaling and its implication in determining cell function and fate. (a) Binding of GF induces dimerization and subsequent phosphorylation of receptor tyrosine kinase (RTK) on the cell membrane. (b) The activated RTK triggers phosphorylation cascades of intracellular kinases. (c) The dynamics (e.g. amplitude, duration, and frequency) of kinase activity determine the biological outputs.

In this work, we present, for the first time, a nongenetic temporal patterning of GF signaling, using a synthetic surrogate of GF. We adopted DNA aptamer as a building block due to its synthetic accessibility and amenability to chemical modifications. Previously, we demonstrated that the aptamer to an RTK $^{6}$ could be used as a synthetic switch, which can control dimerization state of the RTK, depending on external cues ${ }^{7 a}$. The RTK-binding aptamer was designed to form a "sandwich" with a target molecule by tethering the aptamer with an appropriate ligand molecule, thereby enabling receptor dimerization in the presence of the target molecule ${ }^{7 a}$. This concept has been extended to the design of aptamerbased tools for inducing receptor dimerization, responding to other external stimuli, including light irradiation $^{7 b, c}$. For example, Chen et al. reported multi-component system that enables light-induced receptor dimerization using DNA strand exchange reactions, initiated by cleavage of a photo-labile linker ${ }^{7 \mathrm{c}}$. However, none of these tools has realized nongenetic temporal patterning of the GF signaling.

To realize optical control of dynamics of the GF signaling, we designed a single-component system that can turn on its RTK-activating potential, depending on a deprotection event of just one photo- 
(a)

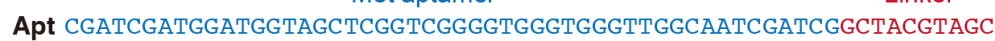
cApt CGATCGATGGATGGTAGCTCGGTCGGGGTGGGTGGGTTGGCAATCGATCGGCTAXGTAGC

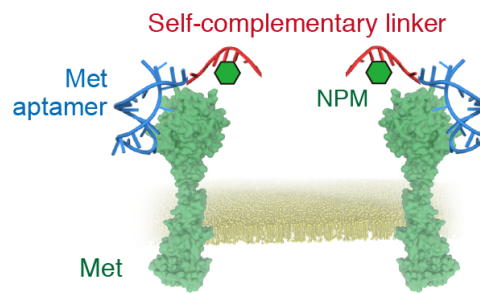

Signal OFF

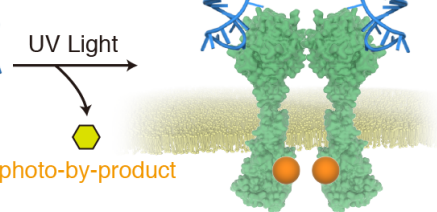

Signal ON

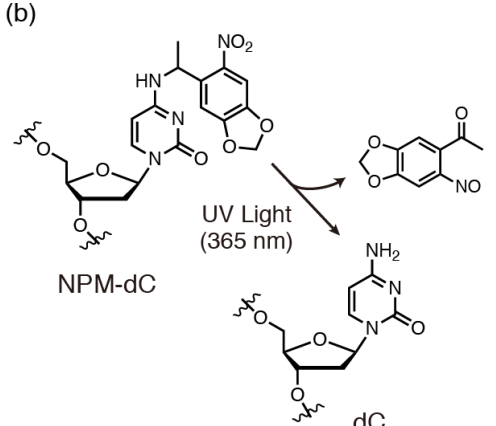

Figure 2. (a) DNA sequences used in the present study and schematic representation of light-induced receptor dimerization with a caged DNA aptamer. The Met-binding aptamer sequence (50-mer) and self-complementary linker sequence (10-mer) are shown in blue and red, respectively. The site of NPM-dC modification is denoted by X. (b) Scheme of the photo-deprotection reaction of NPM$\mathrm{dC}$.

caging group. As a proof-of-concept study, we selected Met, a receptor for hepatocyte growth factor (HGF), as the target ${ }^{8}$. As shown in Figure 2, we designed a single-stranded DNA sequence composed of two functional parts; first, a 50-mer DNA aptamer that binds to Met (blue) $)^{9}$ and a 10-mer selfcomplementary linker sequence (red), tethered to the $3^{\prime}$ terminal of the aptamer sequence. In the linker sequence, 6-nitropiperonyl- $\alpha$-methyl (NPM)-caged deoxycytidine $(\mathrm{dC})^{10}$ was introduced to enable photo-induced dimerization of the aptamer upon irradiation of $365 \mathrm{~nm}$ light (Figure 2). The melting temperature $\left(\mathrm{T}_{\mathrm{m}}\right)$ of the linker sequence with or without the photo-caging group was measured in the physiological conditions (in Dulbecco's phosphate buffered saline). While $\mathrm{T}_{\mathrm{m}}$ value of the linker without the photo-caging was $39.0{ }^{\circ} \mathrm{C}$, the transition in the melting curve was not observed in the case of the linker, which was modified with the NPM-caging group, indicative of significant destabilization of the DNA duplex (Figure S1). Importantly, a clear transition in the melting curve was observed $\left(\mathrm{T}_{\mathrm{m}}=40.7^{\circ} \mathrm{C}\right)$ after an irradiation with 365 nm-peak UV light to the caged linker sequence, suggesting the light-dependent formation of duplex at the physiological conditions. In an assay using Metexpressing DU145 cells, the current system showed excellent off/on ratio in receptor activating potential, as the aptamer was designed to modulate its dimerization propensity, depending on a light irradiation instead of modulating the affinity to the receptor. The

(a)
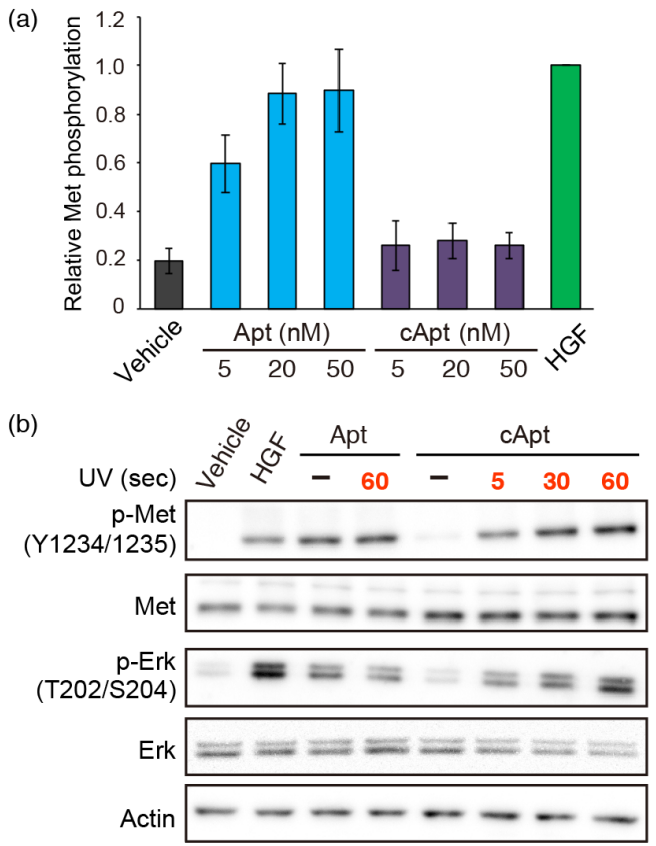

Figure 3. (a) Met phosphorylation induced by HGF (1 $\mathrm{nM})$ or aptamer $(5,20$, and $50 \mathrm{nM})$. DU145 cells were stimulated with the ligand for $15 \mathrm{~min}$ and the resulting Met phosphorylation level was evaluated by ELISA. Error bars indicate SD $(\mathrm{N}=3)$. (b) Western blotting of DU145 cell lysates. The cells were stimulated with HGF (1 nM) or aptamer $(100 \mathrm{nM})$ for $15 \mathrm{~min}$. The samples were irradiated with a $365-\mathrm{nm}$ peak UV irradiation $(8 \mathrm{~mW})$ for the indicated time before the addition to the medium. 
non-caged aptamer (Apt) exhibited increased Met activation level in a concentration dependent manner, while the NPM-caged aptamer (cApt) showed significantly reduced Met activation potential (Figure 3a).

To understand whether a light irradiation can restore the Met activating potential of the cApt, the photo-caged aptamer was irradiated with a 365-nm peak UV light ( $8 \mathrm{~mW}, 5-60 \mathrm{sec})$, before the addition to the culture medium. The result indicated that the cApt showed increased Met-activating potential in an irradiation time-dependent manner (Figure 3b), indicative of production of aptamer dimer upon the liberation of photo-caging group. It was also confirmed that the aptamer could induce activation of the downstream kinase, Erk, after the UV-irradiation.

Finally, we used the caged aptamer for optical control of the dynamics of GF signaling. In the present study, we focused on the dynamics of Erk activity. Previous researches suggested that the dynamics of Erk activity modulate cellular activities, such as proliferation and differentiation ${ }^{2}$. To visualize Erk activity in the living cells, we observed Met-expressing HeLa cells expressing Erk-kinase translocation reporter (KTR)-Clover ${ }^{11}$, a fluorescent reporter for the Erk activity, under time-lapse imaging using a fluorescent microscopy (Figure 4a). This fluorescent reporter was designed to translocate from nucleus to cytosol upon phosphorylation, caused by the activated Erk ${ }^{11}$. Since basal Erk activity and the resulting nuclear localization propensity of the reporter may vary from cell to cell, the cells showing moderate nuclear localization of the reporter at the beginning of the analysis were used for the analysis (Figure S2). We confirmed that the addition of recombinant HGF to the medium induced a prolonged ( $>60 \mathrm{~min})$ translocation of the reporter from nucleus to the cytosol, indicating sustained Erk activation, which was consistent with observation in an immunoblotting analysis (Figure S3).

The cells were incubated in the presence of the cApt $(50 \mathrm{nM})$ and irradiated with a 365-nm peak UV light. When a short pulse $(1 \mathrm{sec})$ of UV irradiation was applied to the cells in 1 min interval for 6 times (input A), translocation of the reporter to the cytosol started to increase within a few minutes (Figures $4 \mathrm{~b}, 4 \mathrm{e}$, and Supplementary video 1). The translocation of reporter reached its maximum after $15 \mathrm{~min}$ from initial irradiation, and decayed to the basal level after another $10 \mathrm{~min}$. This pulse-like Erk activation occurred several minutes after the UV-irradiation and was similar to the observation in the cells, stimulated with a growth factor pulse ${ }^{2 c}$. The translocation of reporter was negligible by the irradiation of the cells in the absence of cApt, thereby discarding the possibility of UV irradiation-dependent activation of the signaling under these experimental conditions (Figure S4).

We found that the dynamics of Erk signaling can be modulated by changing temporal pattern of UV irradiation. When the interval of irradiation pulse was elongated from 1 to 5 min (input B), the reporter showed a prolonged translocation (Figures 4c and 4f). While the duration for the translocation reached a plateau ( $\sim 15 \mathrm{~min})$ was almost comparable to that observed in the cells, stimulated with the irradiation 

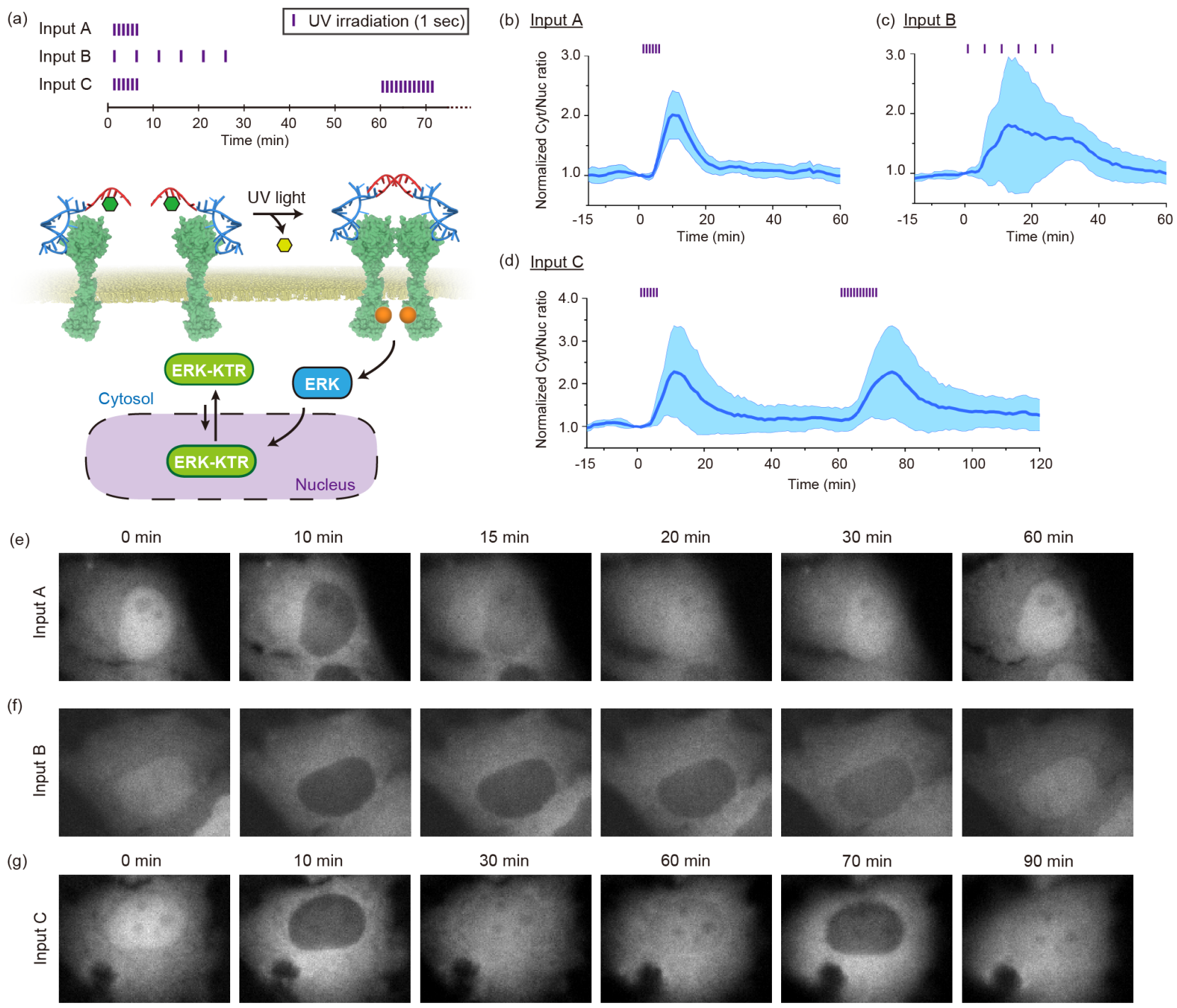

Figure 4. (a) Schematic representation of the experimental workflow. Erk-KTR-Clover is a fluorescence sensor that changes its localization propensity, dependent on the Erk activity. Met activation induces the activation of Erk, thereby inducing the translocation of Erk-KTR-Clover from the nucleus to cytosol. Temporal patterns of the light-irradiation applied to the cells were shown at the top. (b)(d) Normalized ratio of the fluorescent intensity of Erk-KTR-Clover in the cytosol and nucleus. HeLa cells, expressing Erk-KTRClover, were incubated with cApt $(50 \mathrm{nM})$ and a $365-\mathrm{nm}$ peak UV light $\left(0.2 \mathrm{~W} / \mathrm{cm}^{2}\right)$ was irradiated according to the temporal pattern shown in Figure 4a. The data were expressed as average ratio, calculated from 10 cells. The colored areas indicate SD. (e)-(g) Representative time-lapse imaging, showing translocation of Erk-KTR-Clover during time-lapse imaging analysis.

1 pattern A, it took longer time (>30 min) until the translocation level returned to the basal level (Supple-

2 mentary video 2). It was also confirmed that a repetitive UV-irradiation pattern (input C) could induce a

3 pulsatile Erk activation pattern (Figures 4d, 4g and Supplementary video 3).

We also elucidated spatial resolution of the current system. The irradiation pattern A (Figure 4a) was applied to a selected area and the translocation of reporter in the cells, either inside or outside of the

6 irradiated area, was monitored (Figure S5 and Supplementary video 4). In the cells located in the irradi-

7 ated area, Erk-reporter showed a pulse-like translocation pattern when the cells were stimulated with the

8 irradiation scheme A. On the contrary, the translocation of reporter was negligible in the cells outside of 
the area. These results indicate that activation of Met receptor occurred only in the light-irradiated area and suggest that the influence of diffusion of uncaged aptamers was negligible. This excellent spatial resolution is presumably due to the excess amount of uncaged aptamers, existing in the non-irradiated area, which can compete for the receptor binding with the active aptamer produced in the irradiated area.

To summarize, we demonstrated for the first time, nongenetic temporal patterning of GF signaling using a non-proteinaceous growth factor surrogate. The caged DNA aptamer to RTK could control the dynamics of GF signaling with a temporal precision on the minute timescale. Unlike precedent optochemical tools, based on a combination of chemically-inducible dimerization protein, and an appropriate photo-caged dimerizer ${ }^{12}$, this nongenetic approach applies solely a photocaged-aptamer that can directly control the protein dimerization on the living cells. Although other excellent DNA aptamer-based tools for optical control of receptor dimerization have been recently reported ${ }^{7 b, c}$, the current system has significant advantage. The aptamer was designed to turn on its receptor-activating potential, depending on a deprotection event of just one photo-caging group. This feature enables controlling of the GF signaling with better spatio-temporal precision over the precedent system, utilizing a DNA strand exchange reaction $^{7 \mathrm{c}}$ or release of agonist DNA from nanoparticles ${ }^{7 \mathrm{~b}}$. This novel class of optochemical tools represents a promising approach for studying the association between signaling dynamics and resulting biological outcomes, aiming to manipulate cellular functions and fate of living cells.

\section{ACKNOWLEDGMENT}

pENTR-ERKKTRClover was a gift from Dr. Markus Covert (Addgene plasmid \#59138). This work was supported by Core Research for Evolutional Science and Technology (CREST) of Molecular Technology (\#JPMJCR13L4) from Japan Science and Technology Agency (JST) for S.S, a Grant-in-Aid for Young Scientists (\#19K15693) from Japan Society for the Promotion of Science (JSPS), a Grant for Basic Science Research Projects from the Sumitomo Foundation, and Noguchi-Shitagau Research Grant from the Noguchi Institute for R.U.

\section{AUTHOR INFORMATION}

Corresponding Authors

*r.ueki@chembio.t.u-tokyo.ac.jp

*ssando@chembio.t.u-tokyo.ac.jp

\section{REFERENCES}

3 (1) Lemmon, M. A.; Schlessinger, J. Cell 2010, 141, 1117. 
(2) (a) Wilson, M. Z.; Ravindran, P. T.; Lim, W. A.; Toettcher, J. E. Mol. Cell 2017, 67, 757. (b) Sparta, B.; Pargett, M.; Minguet, M.; Distor, K.; Bell, G.; Albeck, J. G. J. Biol. Chem. 2015, 290, 24784. (c) Ryu, H.; Chung, M.; Dobrzyński, M.; Fey, D.; Blum, Y.; Lee, S. S.; Peter, M.; Kholodenko, B. N.; Jeon, N. L.; Pertz, O. Mol. Syst. Biol. 2015, 11, 838. (d) Albeck, J. G.; Mills, G. B.; Brugge, J. S. Mol. Cell 2013, 49, 249. (e) Toettcher, J. E.; Weiner, O. D.; Lim, W. A. Cell 2013, 155, 1422.

(3) Marshall, C. J. Cell 1995, 80, 179.

(4) (a) Duan, L.; Hope, J. M.; Guo, S.; Ong, Q.; François, A.; Kaplan, L.; Scherrer, G.; Cui, B. ACS Synth. Biol. 2018, 7, 1685. (b) Kainrath, S.; Stadler, M.; Reichhart, E.; Distel, M.; Janovjak, H. Angew. Chem. Int. Ed. 2017, 56, 4608. (c) Reichhart, E.; Ingles-Prieto, A.; Tichy, A. M.; McKenzie, C.; Janovjak, H. Angew. Chem. Int. Ed. 2016, 55, 6339. (d) Chang, K. Y.; Woo, D.; Jung, H.; Lee, S.; Kim, S.; Won, J.; Kyung, T.; Park, H.; Kim, N.; Yang, H. W.; Park, J. Y.; Hwang, E. M.; Kim, D.; Heo, W. D. Nat. Commun. 2014, 5, 4057. (e) Grusch, M.; Schelch, K.; Riedler, R.; Reichhart, E.; Differ, C.; Berger, W.; Inglés-Prieto, Á; Janovjak, H. EMBO J. 2014, 33, 1713. (f) Kim, N.; Kim, J. M.; Lee, M.; Kim, C. Y.; Chang, K. Y.; Heo, W. D. Chem. Biol. 2014, 21, 903.

(5) Miller, D. S.; Chirayil, S.; Ball, H. L.; Luebke, K. J. ChemBioChem 2009, 10, 577.

(6) Ueki, R.; Ueki, A.; Kanda, N.; Sando, S. Angew. Chem. Int. Ed. 2016, 55, 579.

(7) (a) Ueki, R.; Atsuta, S.; Ueki, A.; Sando, S. J. Am. Chem. Soc. 2017, 139, 6554. (b) Wang, M.; He, F.; Li, H.; Yang, S.; Zhang, J.; Ghosh, P.; Wang, H. H.; Nie, Z. Nano Lett. 2019, 19, 2603. (c) Chen, S.; Li, J.; Liang, H.; Lin, X. H.; Li, J.; Yang, H. H. Chem. Eur. J. 2018, 24, 15988.

(8) (a) Trusolino, L.; Bertotti, A.; Comoglio, P. M. Nat. Rev. Mol. Cell Biol. 2010, 11, 834. (b) Birchmeier, C.; Birchmeier, W.; Gherardi, E.; Vande Woude, G. F.; Nat. Rev. Mol. Cell Biol. 2003, 4, 915. (9) (a) Ueki, R.; Sando, S. Chem. Commun. 2014, 50, 13131. (b) Boltz, A.; Piater, B.; Toleikis, L.; Guenther, R.; Kolmar, H.; Hock, B. J. Biol. Chem. 2011, 286, 21896.

(10) Govan, J. M.; Uprety, R.; Hemphill, J.; Lively, M. O.; Deiters, A. ACS Chem. Biol. 2012, 7, 1247.

(11) Regot, S.; Hughey, J. J.; Bajar, B. T.; Carrasco, S.; Covert, M. W. Cell 2014, 157, 1724.

(12) (a) Brown, K. A.; Zou, Y.; Shirvanyants, D.; Zhang, J.; Samanta, S.; Mantravadi, P. K.; Dokholyan, N. V.; Deiters, A. Chem. Commun. 2015, 51, 5702. (b) Schelkle, K. M.; Griesbaum, T.; Ollech, D.; Becht, S.; Buckup, T.; Hamburger, M.; Wombacher, R. Angew. Chem. Int. Ed. 2015, 54, 2825. (c) Ballister, E. R.; Aonbangkhen, C.; Mayo, A. M.; Lampson, M. A.; Chenoweth, D. M. Nat. Commun. 2014, 5, 5475. (d) Zimmermann, M.; Cal, R.; Janett, E.; Hoffmann, V.; Bochet, C. G.; Constable, E.; Beaufils, F.; Wymann, M. P. Angew. Chem. Int. Ed. 2014, 53, 4717. (e) Umeda, N.; Ueno, T.; Pohlmeyer, C.; Nagano, T.; Inoue, T. J. Am. Chem. Soc. 2011, 133, 12. (f) Karginov, A. V.; Zou, Y.; 
1 Shirvanyants, D.; Kota, P.; Dokholyan, N. V.; Young, D. D.; Hahn, K. M.; Deiters, A. J. Am. Chem. 2 Soc. 2011, 133, 420. 
Nongenetic Control of Receptor Signaling Dynamics with a DNA-based Optochemical Tool

Ryosuke Ueki, ${ }^{* \ngtr}$ Shota Hayashi, ${ }^{\dagger}$ Masaya Tsunoda, ${ }^{\dagger}$ Momoko Akiyama, ${ }^{\dagger}$ Hanrui Liu, Tasuku Ueno, ${ }^{*}$ Yasuteru Urano, ${ }^{*}, \&$ and Shinsuke Sando ${ }^{*}$, ,

tDepartment of Chemistry and Biotechnology and "Department of Bioengineering, The University of Tokyo, 7-3-1 Hongo, Bunkyo-ku, Tokyo, 113-8656, Japan.

${ }^{*}$ Graduate School of Pharmaceutical Sciences and ${ }^{\S}$ Graduate School of Medicine, The University of Tokyo, 7-3-1 Hongo,

Bunkyo-ku, Tokyo 113-0033, Japan.

*e-mail:r.ueki@chembio.t.u-tokyo.ac.jp; ssando@chembio.t.u-tokyo.ac.jp

\section{Table of Contents}

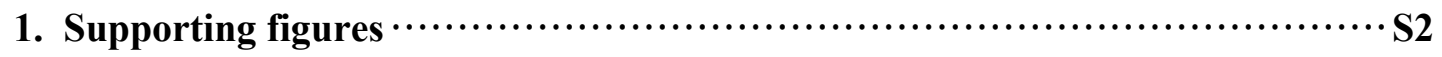

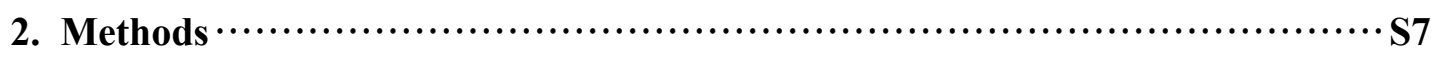

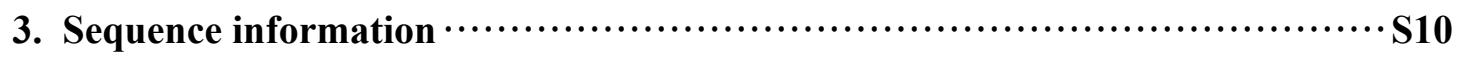

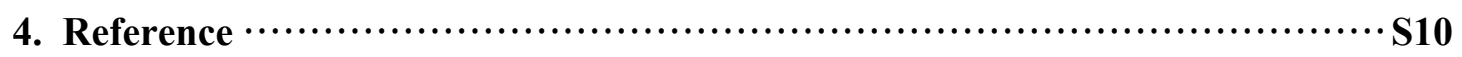




\section{Supporting figures}
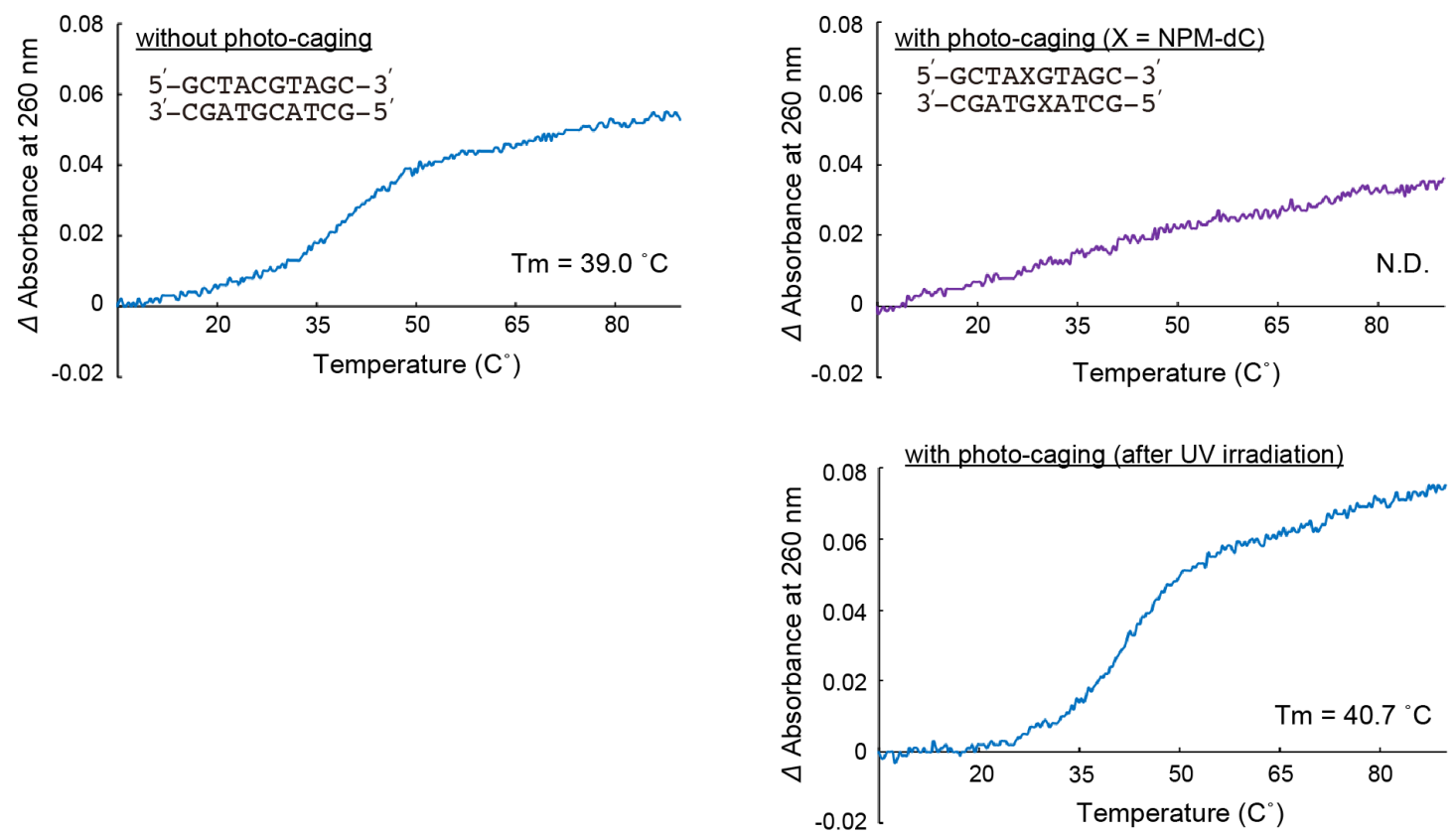

Figure S1. Melting curves of the linker sequence of Apt (2.5 $\mu \mathrm{M}$, top left $)$ or $\mathrm{cApt}(2.5 \mu \mathrm{M}$, top right) in DPBS. The linker sequence of cApt was irradiated with $365 \mathrm{~nm} \mathrm{UV} \mathrm{light}(8 \mathrm{~mW}, 60 \mathrm{sec})$ and used for the melting curve analysis (bottom, right). 


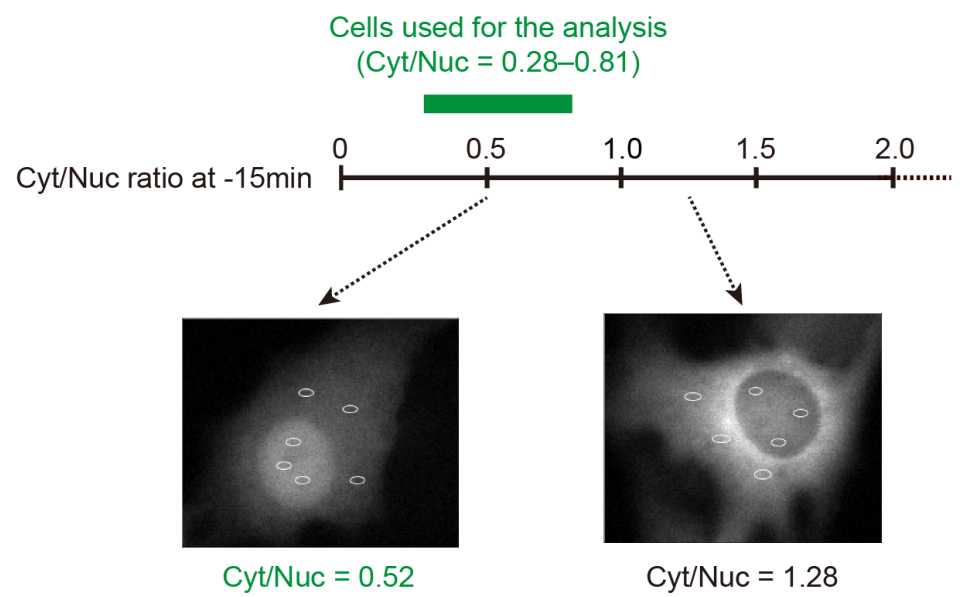

Figure S2. Representative fluorescence images showing expression of Erk-KTR-Clover in HeLa cells. The cytoplasmic versus nuclear fluorescence of the Erk-KTR-Clover (Cyt/Nuc ratio) in each cell was calculated using average nuclear or cytoplasmic fluorescent intensities, determined from three randomly selected ROIs (white circles) in a background-subtracted image. To limit the effects of variability in basal Erk activity of each cell, the cells with Cyt/Nuc ratio between 0.28 and 0.81 at the beginning of time-lapse imaging $(t=-15 \mathrm{~min})$ were randomly selected and used for the analysis. 


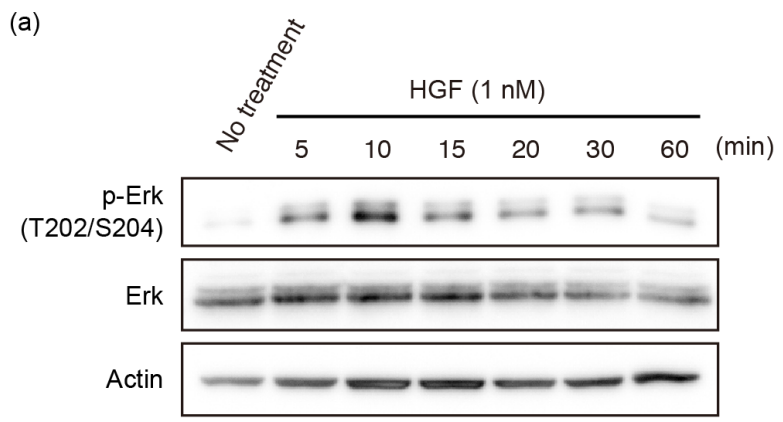

(b)
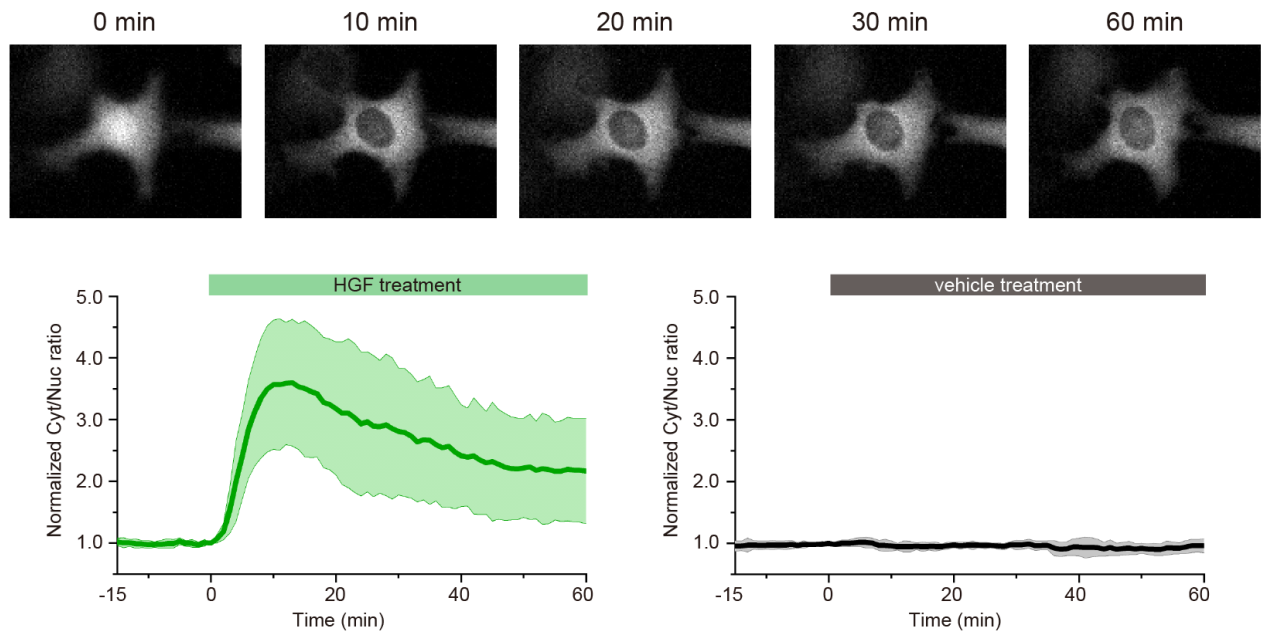

Figure S3. (a) Immunoblotting analysis of Erk phosphorylation kinetics in HeLa cells, treated with HGF (1 nM). (b) Time-lapse imaging of translocation of Erk-KTR-Clover in HeLa cells, treated with HGF (1 nM) or vehicle control (DPBS) for $60 \mathrm{~min}$. The data are expressed as average ratio from eight cells. The colored areas indicate SD. 

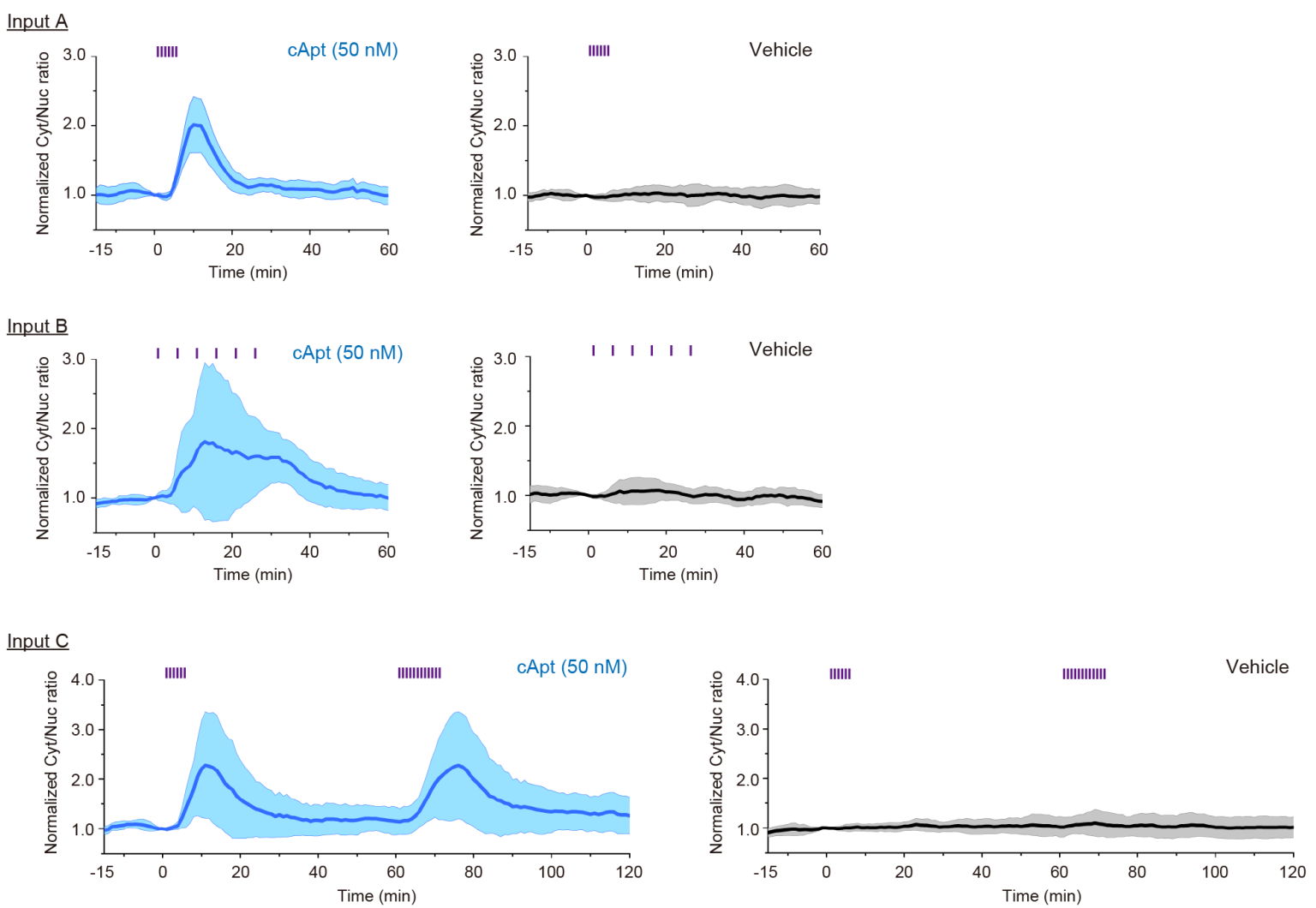

Figure S4. Normalized ratio of the fluorescent intensity of Erk-KTR-Clover in the cytosol and nucleus. HeLa cells, expressing Erk-KTR-Clover, were incubated in the absence (gray) or presence (blue) of cApt (50 nM) and a series of $365 \mathrm{~nm}$ UV irradiations (purple bars). The data are expressed as average ratio from 10 cells. The colored areas indicate SD. 
(a)

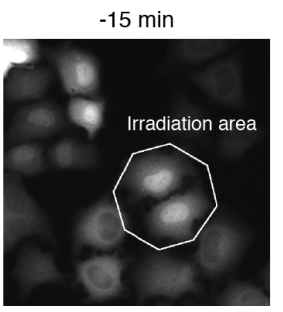

$0 \mathrm{~min}$

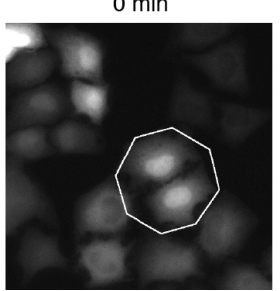

(b)

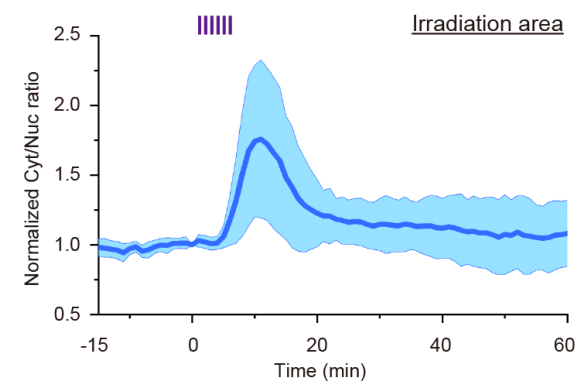

$10 \mathrm{~min}$

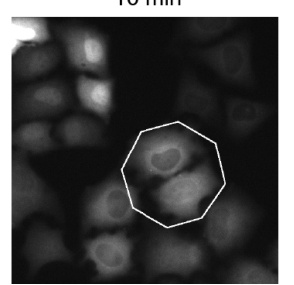

$20 \mathrm{~min}$

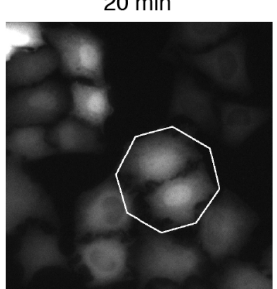

IIIIII

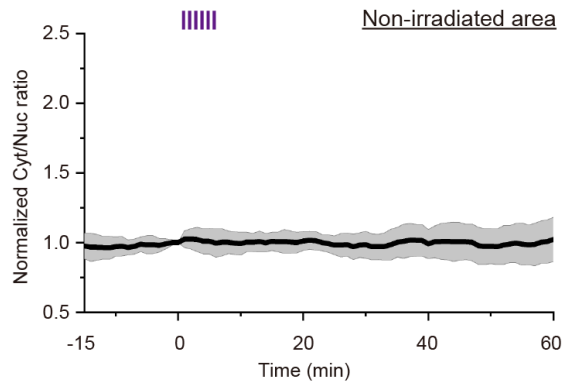

$30 \mathrm{~min}$

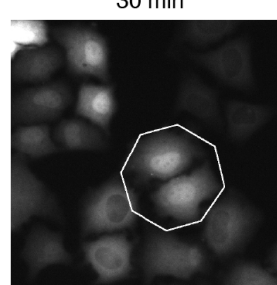

Figure S5. (a) Representative time-lapse imaging showing translocation of Erk-KTR-Clover. The irradiation pattern A (Figure 4a) was applied to the octagonal area (white) and the translocation of reporter in the cells, inside or outside of the irradiation area, was monitored. (b) Normalized ratio of the fluorescent intensity in the cytosol and nucleus. HeLa cells, expressing Erk-KTR-Clover, were incubated in the presence of cApt $(50 \mathrm{nM})$ and lightirradiated with a temporal pattern A shown in Figure 4a. The data are expressed as average ratio and the colored areas indicate $\mathrm{SD}$ for the irradiated $(\mathrm{N}=7)$ and the non-irradiated $(\mathrm{N}=16)$ areas. 


\section{Methods}

\section{2-1 General information}

Reagents were purchased from the standard suppliers and used without further purification. All oligonucleotides were purchased from Fasmac and Gene design and used without further purification. The cApt sequence was synthesized by Gene design, using 6-nitropiperonyl- $\alpha$-methyl (NPM)-caged deoxycytidine $(\mathrm{dC})$ phosphoramidite. The NPM-caged $\mathrm{dC}$ phosphoramidite was synthesized according to the literature ${ }^{1}$. Refolding and annealing of DNA samples were performed with a thermal cycler. A xenon light source (MAX-301, Asahi Spectra, Tokyo, Japan) was used for uncaging experiments, shown in Figures $3 b$ and S1. Fluorescent images were obtained with an inverted microscope (IX-81, Olympus, Tokyo, Japan), equipped with a sCMOS camera (Zyla4.2, Andor technology, Belfast, Northern Ireland). The filter cube U-MWIB3 and U-MNU2 (Olympus, Tokyo, Japan) were used for the detection of ErkKTR-Clover and uncaging of the caged-nucleotide, respectively. Immunoblotting images were obtained with a luminescence detection system (AE-9300H, ATTO, Tokyo, Japan). Absorbance and fluorescence measurements were performed with Infinite M200 pro (Tecan, Bezirk Meilen, Switzerland). UV melting measurement was performed with UV-VIS spectrometer (UV-1650PC, Shimadzu, Kyoto, Japan).

\section{2-2 Cell culture}

DU145 cells and HeLa cells were cultured in RPMI1640 supplemented with $10 \%$ fetal bovine serum (FBS) and 1\% antibiotic-antimycotic. All cells were maintained at $5 \% \mathrm{CO}_{2}$ in a humidified incubator at $37^{\circ} \mathrm{C}$.

\section{2-3 Preparation of cell lysates}

The cells were seeded onto $35 \mathrm{~mm}$ dishes and cultured overnight at $5 \% \mathrm{CO}_{2}$ in a humidified incubator at $37^{\circ} \mathrm{C}$. The cells were then starved for $24 \mathrm{~h}$ in RPMI1640 without FBS. After the starvation, the medium was replaced and the cells were stimulated with an appropriate ligand for $15 \mathrm{~min}$ at $37{ }^{\circ} \mathrm{C}$. The ligand solution was pre-warmed at $37^{\circ} \mathrm{C}$ just before the addition to the medium. The UV light was irradiated to the ligand solution that was kept at $37^{\circ} \mathrm{C}$ on the thermal cycler for an appropriate time. Next, the cells were washed twice with DPBS and lysed with a lysis buffer (20 mM Tris- $\mathrm{HCl} \mathrm{pH} 7.5,150 \mathrm{mM} \mathrm{NaCl}, 1$ 
mM EDTA, $2.5 \mathrm{mM}$ sodium pyrophosphate, $1 \mathrm{mM} \beta$-glycerophosphate, $1 \mathrm{mM}$ sodium orthovanadate, 1 $\mathrm{mM}$ AEBSF, $1 \mu \mathrm{g} / \mathrm{mL}$ leupeptin, $1 \%$ Triton X-100, and 10\% glycerol). The cell lysates were centrifuged at $10,000-11,000 \times \mathrm{g}$ for $20 \mathrm{~min}$ and then the supernatants were recovered. The total protein concentration of each cell lysate was measured with the standard BCA assay after the dilution.

\section{2-4 ELISA}

The detection of phosphorylated Met was performed using the Human Phospho-HGFR/c-MET DuoSet IC ELISA (DYC2480-2, R\&D systems, Minneapolis, USA). The assay was performed according to the manufacturer's instructions. The normalized Met phosphorylation level of each cell lysate was calculated by dividing the absorbance of the assay at $450 \mathrm{~nm}$ to the total protein concentration of the lysate. Three independent experiments were performed and the mean values were indicated with error bars (SD). The data were expressed as relative values, compared with the normalized Met phosphorylation level, induced by the treatment with HGF $(1 \mathrm{nM})$ for 15 min in each experiment.

\section{2-5 Immunoblotting assay}

The cell lysates were separated by SDS-PAGE and then transferred to a PVDF membrane. The membrane was incubated with a primary antibody at $4{ }^{\circ} \mathrm{C}$ overnight, following the incubation with an appropriate secondary antibody at room temperature for $1 \mathrm{~h}$. The primary antibodies for phospho-Met (3077), Met (8198), phospho-ERK1/2 (4370), ERK1/2 (4695, figure 3b) and beta-actin (4967) were obtained from Cell Signaling Technology (Danvers, USA). The primary antibody for ERK1/2 (sc-514302, figure S3) was obtained from Santa Cruz Biotechnology (Dallas, USA). The Anti-Rabbit Immunoglobulins/HRP secondary antibody was obtained from Agilent Technologies (P0488, Santa Clara, USA). The AntiMouse Immunoglobulins/HRP secondary antibody (W4021) was obtained from Promega (Madison, USA). The membranes were probed using ImmunoStar LD (296-69901, FUJIFILM Wako Pure Chemical Corporation, Tokyo, Japan) or Immobilon Western Chemiluminescent HRP Substrate (WBKLS0500, Merck Millipore, Burlington, USA). 


\section{2-6 UV melting measurement}

UV melting measurement was performed with UV-VIS spectrometer (UV-1650PC, Shimadzu, Kyoto, Japan) over a temperature of $5-90{ }^{\circ} \mathrm{C}$ (heat rate $\left.=0.5^{\circ} \mathrm{C} / \mathrm{min}\right)$ using a quartz cuvette. DNA $(2.5 \mu \mathrm{M})$ was dissolved in Dulbecco's phosphate buffered saline and refolded with a thermal cycler $\left(95^{\circ} \mathrm{C}\right.$ for 5 min, and then cooled to $4{ }^{\circ} \mathrm{C}$ ) before measurements. The UV melting curve was obtained by subtracting the background absorbance at $320 \mathrm{~nm}$ from the absorbance at $260 \mathrm{~nm}$. Two independent experiments were performed and the mean Tm values were indicated.

\section{2-7 Preparation of Erk-KTR-Clover expressing cells}

An episomal vector encoding Erk-KTR-Clover was constructed for time-lapse imaging of Erk-KTRClover translocation. The Erk-KTR-Clover sequence was amplified from pENTR-ERKKTR Clover plasmid (Addgene 59138) and inserted into pEBMulti-Puro vector. The sequence of the constructed vector was confirmed by Sanger sequencing. HeLa cells were seeded at $60 \mathrm{~mm}$-plates and cultured in RPMI1640 supplemented with 10\% FBS overnight. On the day of transfection, the cells were washed twice with DPBS and incubated with DNA/PEI mixture in RPMI1640 supplemented with 10\% FBS overnight. To enrich Erk-KTR-Clover expressing cells, drug selection $(1 \mathrm{mg} / \mathrm{ml}$ puromycin in RPMI1640 supplemented with $10 \%$ FBS) was started on the next day after the transfection. The cells were used for time-lapse imaging of Erk-KTR-Clover translocation at least 2 days after the drug selection.

\section{2-8 Time-lapse imaging of Erk-KTR-Clover translocation}

The Erk-KTR-Clover-expressing HeLa cells $\left(2 \times 10^{4}\right.$ cells/well $)$ were seeded onto a chambered coverglass (8 wells, AGC Techno Glass, Tokyo, Japan) and cultured in RPMI1640 supplemented with $10 \%$ FBS. After the cells adhered to the coverglass, they were incubated in a starving medium (RPMI1640 without phenol red) overnight. On the day of experiment, the media was replaced and the chambered coverglass was mounted on a stage top incubator (INUG2F-IX3W, Tokai Hit, Shizuoka, Japan). The cells were incubated at $5 \% \mathrm{CO}_{2}$ in a humidified atmosphere at $37{ }^{\circ} \mathrm{C}$ during the experiment. For uncaging experiments, the cells were pre-incubated in the presence or absence of cApt $(50 \mathrm{nM})$ in the 
starving medium at least for $30 \mathrm{~min}$. Time-lapse images were captured in $1 \mathrm{~min}$ intervals for $75 \mathrm{~min}$ (Figures 4b, 4c, 4e, 4f, S3, S5), 135 min (Figures 4d and 4g). The filter cubes U-MWIB3 and U-MNU2 (Olympus, Tokyo, Japan) were used for detection of Erk-KTR-Clover and for photo-deprotection of the caged-nucleotide, respectively. The Iris diaphragm was used for local UV irradiation. The location of UV irradiation at the sample plane was checked by observing Erk-KTR-Clover-expressing HeLa cells, cultured on the chambered coverglass in a high confluency. Both global and local irradiations were performed for the indicated duration while conducting time-lapse imaging. The cytoplasmic versus nuclear fluorescence of the Erk-KTR-Clover (Cyt/Nuc ratio) in each cell was calculated using average nuclear or cytoplasmic fluorescent intensities, determined from three randomly selected ROIs in a background-subtracted image. To limit the effects of variability in basal Erk activity of each cell, the cells with Cyt/Nuc ratio between 0.28 and 0.81 at the beginning of time-lapse imaging $(t=-15 \mathrm{~min})$ were randomly selected and used for the analysis. The image analysis was conducted using Image $\mathrm{J}$ software (http://rsbweb.nih.gov/ij/).

\section{Sequence data}

Apt: CGA TCG ATG GAT GGT AGC TCG GTC GGG GTG GGT GGG TTG GCA ATC GAT CGG CTAC GTA GC

cApt: CGA TCG ATG GAT GGT AGC TCG GTC GGG GTG GGT GGG TTG GCA ATC GAT CGG CTAX GTA GC

(X = NPM-caged deoxycytidine. Hybridization linker sequences are highlighted in red)

\section{Reference}

1. Govan, J. M.; Uprety, R.; Hemphill, J.; Lively, M. O.; Deiters, A. ACS Chem. Biol. 2012, 7, 1247. 


\section{Other files}

Supporting video 1.avi (1.80 MiB)

Supporting video 2.avi (1.93 MiB)

Supporting video 3.avi (3.22 MiB)

Supporting video 4.avi (2.33 MiB) view on ChemRxiv • download file view on ChemRxiv • download file view on ChemRxiv • download file view on ChemRxiv - download file 